\title{
Geometric calibration of the SND detector electromagnetic calorimeter
}

\author{
A.A. Korol ${ }^{a, b}$, N.A. Melnikova ${ }^{a, *}$ \\ ${ }^{a}$ Budker Institute of Nuclear Physics, Novosibirsk, 630090, Russia \\ ${ }^{b}$ Novosibirsk State University, Novosibirsk, 630090, Russia
}

\begin{abstract}
This paper presents the design, implementation and validation of the software alignment procedure used to perform geometric calibration of the electromagnetic calorimeter with respect to the tracking system of the SND detector which is taking data at the VEPP-2000 $e^{+} e^{-}$collider (BINP, Novosibirsk). This procedure is based on the mathematical model describing the relative calorimeter position. The parameter values are determined by minimizing a $\chi^{2}$ function using the difference between particle directions reconstructed in these two subdetectors for $e^{+} e^{-} \rightarrow e^{+} e^{-}$scattering events. The results of the calibration applied to data and MC simulation fit the model well and give an improvement in particle reconstruction. They are used in data reconstruction and MC simulation.
\end{abstract}

Keywords: particle detector, electromagnetic calorimeter, alignment, calibration

PACS: 29.40.Vj, 06.60.Sx, 07.05.-t

\section{Introduction}

The Spherical Neutral Detector (SND) [1, 2, 3] is used at the electronpositron collider VEPP-2000 [4] for hadronic cross-section measurement in the center of mass energy range $0.3 \div 2.0 \mathrm{GeV}$. The detector consists of several subsystems (Fig (1) including a spherical electromagnetic calorimeter (EMC) and a cylindrical tracking system (TS). The detector also features a threshold Cherenkov counters and a muon detector.

\footnotetext{
${ }^{*}$ Corresponding author

Email address: n.a.melnikova@inp.nsk.su (N.A. Melnikova)
} 

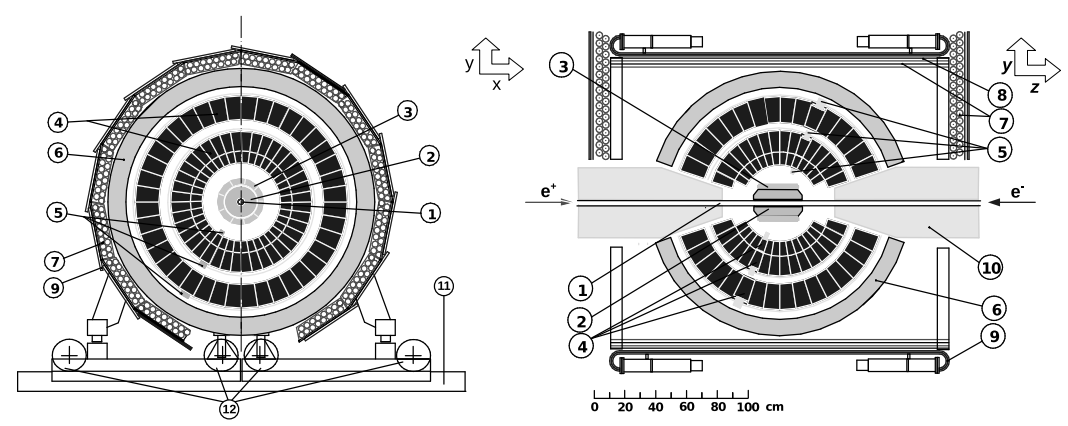

Figure 1: The SND scheme: 1 - vacuum pipe, 2 - tracking system (TS), 3 - threshold Cherenkov counter, 4-5 - electromagnetic calorimeter (NaI (Tl)) (EMC), 6 - iron absorber, 7-9 - muon detector, 10 - focusing solenoids, 11 - rails, 12 - wheels.

The EMC is composed of $1632 \mathrm{NaI}(\mathrm{TI})$ crystal counters arranged in 3 spherical layers. Its spherical shape covers the polar angle range from $18^{\circ}$ to $162^{\circ}$ and provides uniform particle detection for $95 \%$ of the total solid angle. The TS is a 9-layer drift chamber with an axial position measurement using the cathode strips and charge division on the wires. The EMC and the TS provide the main information for event reconstruction: the TS measures parameters of charged particle trajectories $\left(z_{0}, d_{0}, \varphi_{T S}, \theta_{T S}\right)$, while the EMC measures the energy and the angular position of electromagnetic showers $\left(\varphi_{E M C}, \theta_{E M C}\right)$. To correctly reconstruct physical events in the detector it is important to know precise positions of the EMC counters relative to the TS. However data demonstrates that the EMC is misaligned as shown at Fig 2 ,

For the most interesting classes of charged particles ( $\pi$ - and K-mesons) only the TS track information is relevant. On the other hand, photons, which are in particular used to make $\pi^{0}$ and $\eta$ meson candidates, are reconstructed only by electromagnetic shower energy distribution in the EMC, inferring their direction from the assumed center of the calorimeter. Consequently, even small misalignments of the EMC and TS can be a source of kinematic inconsistency for data analysis. Furthermore the EMC consists of two separate hemispheres and space angles between photons could be also skewed. Fortunately, electrons have both tracks in the TS and electromagnetic showers in the EMC. So, we use $e^{+} e^{-} \rightarrow e^{+} e^{-}$events to measure the EMC position relative to the TS.

Different alignment solutions are well described in the literature for homogeneous multicomponent detectors (e.g. vertex detectors). Techniques based on track residual minimization are usually used for tracking detectors. These methods minimize distance between the measured hits and the corresponding reconstructed hits determining the detector element position in space. Such algorithms are being used for the ATLAS, CMS, LHCb detectors (LHC collider), for the STAR detector (RHIC collider), for the BaBar detector (PEP-II collider), for the $\mathrm{H} 1$ detector (HERA collider) etc. [5]. However, there is a lack of published results for heterogeneous systems (like EMC vs TS). While such calibration studies were definitely conducted for the ATLAS [6], KEDR [7], 


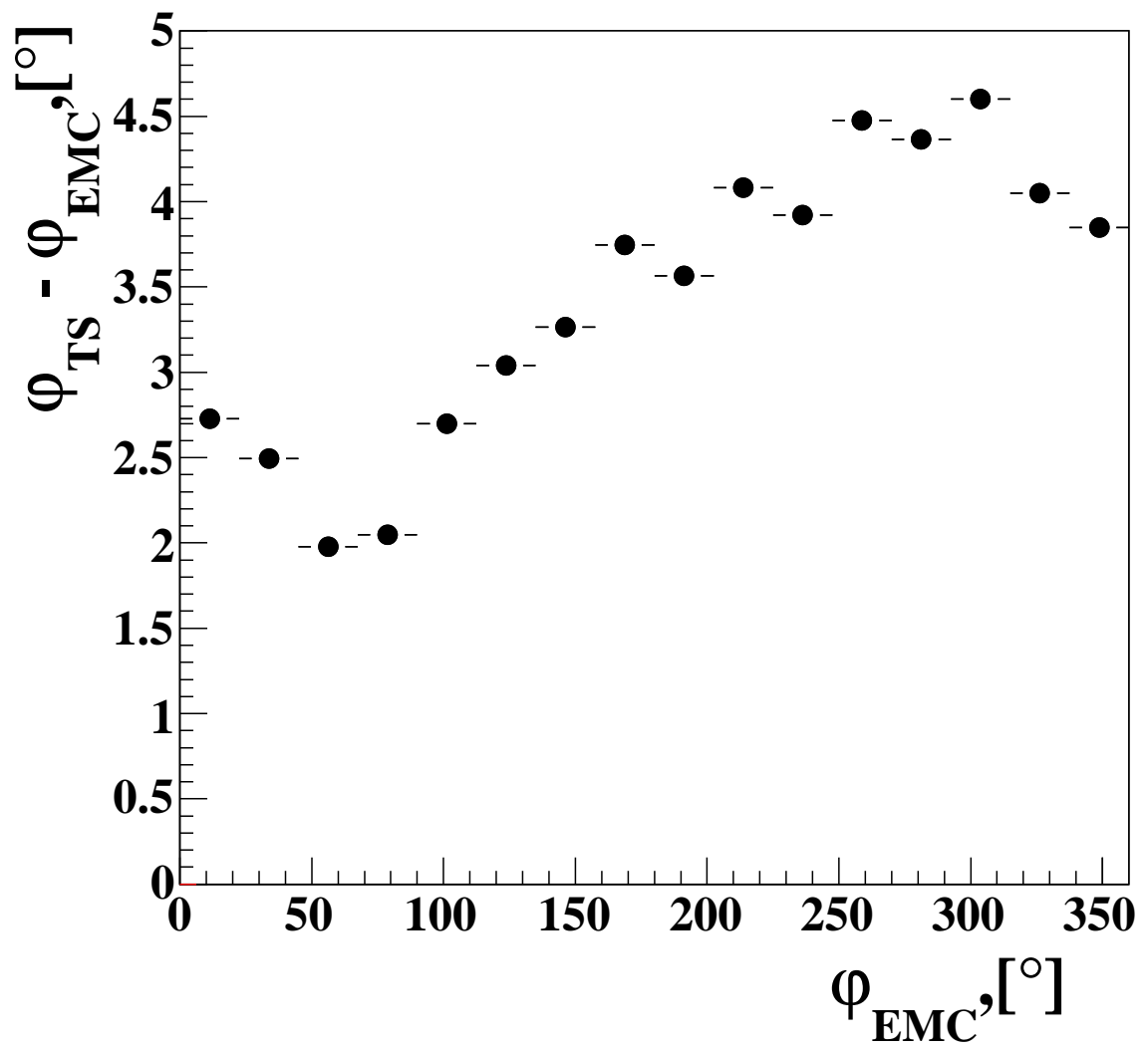

Figure 2: The difference between the azimuthal angles reconstructed in the TS and in the EMC for $e^{+} e^{-} \rightarrow e^{+} e^{-}$events. 
CMD-3 [8], BaBar [9] experiments (as we know from private communications) they were not openly documented. The complication here is different sets of reconstructed parameters for subdetectors: a 5-parameter helix (or a 4-parameter line in space in our case) for various tracking systems versus 2-parameter shower direction from the center reconstructed in the EMC calorimeters.

\section{Calorimeter position parametrization}

The EMC counters are rigidly fixed to two supporting aluminium spheres: the first is for the two inner layers of counters, while the second is for the third layer. In addition, the EMC is divided into two halves, so that when disassembling the SND these two hemispheres can be easily moved in the horizontal direction orthogonal to the detector axis using rails and wheels (as illustrated on Fig 1 labels 11 and 12). This model of assembling and disassembling the EMC allows to achieve a position accuracy of $3 \div 5 \mathrm{~mm}$. To improve it the software geometric calibration procedure is performed using $e^{+} e^{-} \rightarrow e^{+} e^{-}$events reconstructed both in the EMC and in the TS.

The mathematical model of the EMC sensitive element (counter) positions relative to the TS considers the EMC halves as rigid bodies. Counter positions take into account a possible EMC misalignment as a whole relative to the TS and relative misalignments of the EMC halves. As the counters are rigidly fixed to the supporting spheres with the position accuracy of not worse than $0.03 \mathrm{~cm}$ individual displacement of each counter is small to be ignored and not considered here.

We measure the EMC misalignment relative to the TS using angular differences $\sin \left(\varphi_{T S, R}-\varphi_{E M C}\right)$ and $\theta_{T S, R}-\theta_{E M C}$, where $\varphi_{T S, R}$ and $\theta_{T S, R}$ represent the expectation for the EMC cluster direction from the TS track. They are calculated as the polar coordinates of the point $\boldsymbol{p}_{\boldsymbol{R}}$ (Fig (3), in which the track crosses a sphere with the radius

$$
R=R_{0}+X_{0}\left(x_{\max }-\frac{x_{\text {pre }}}{\sin \theta_{T S}}\right),
$$

where $R_{0}$ is the EMC inner radius, $X_{0}=2.56 \mathrm{~cm}$ is the radiation length for $\mathrm{NaI}, E_{c}=13.16 \mathrm{MeV}$ is its critical energy, $x_{\max }=\ln \frac{E}{E_{c}}-0.5$ is the maximum of the longitudinal electromagnetic shower distribution [10], $x_{\text {pre }} \approx 0.13$ is an estimation of the thickness of material between the interaction point and the EMC (0.04 in the TS [11] , and 0.09 in the Cherenkov counters [12]).

Unfortunately, the maximum of the energy deposition in the EMC is located in the first two layers, making it impossible to estimate the misalignment of the third layer.

The right handed TS coordinate system is used as a reference coordinate system with center at the TS center and the $\mathrm{Z}$ axis is directed along the TS axis. The $\mathrm{Y}$ axis is directed vertically upwards, while the $\mathrm{X}$ axis is directed to the collider ring center. The EMC coordinate system without misalignment is the same. 


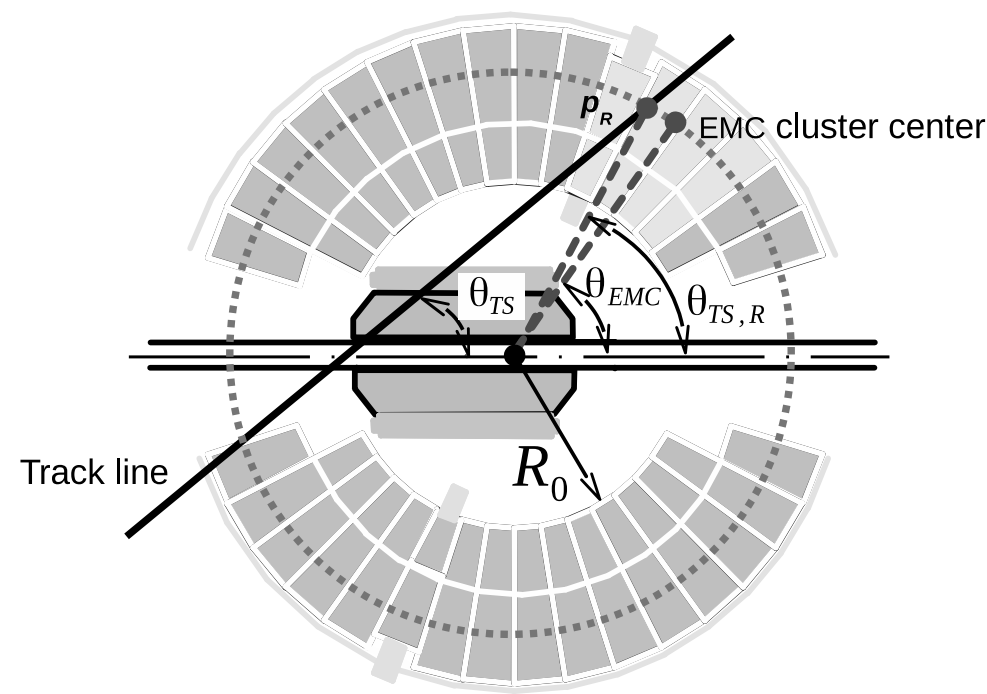

Figure 3: Effective shower position in the EMC reference system. The notations is described in the main text.

To describe the EMC position relative to the TS, two sets of parameters are used. The first set describes the rotation and the shift of the EMC as a whole:

1. $\alpha$ is the angle of the EMC rotation around the TS Z axis $\left(0^{\circ}<\alpha<360^{\circ}\right)$;

2. $\beta$ is the EMC tilting angle with respect to the TS $\mathrm{Z}$ axis $\left(0^{\circ}<\beta<90^{\circ}\right)$;

3. $\psi$ is a direction of the $\beta$ tilting $\left(0^{\circ}<\psi<180^{\circ}\right)$;

4. $d x, d y, d z$ are the EMC shifts in corresponding directions relative to the TS.

The second set of parameters describes rotations and shifts of the hemispheres relative to the EMC (Fig 4):

1. $\mu$ is the half-angle of the EMC hemispheres separation $\left(0^{\circ}<\mu<90^{\circ}\right)$;

2. $\tau$ is the separation direction angle from the vertical direction $\left(-180^{\circ}<\tau<180^{\circ}\right)$;

3. $d x_{r e l}, d y_{r e l}, d z_{r e l}$ are the hemisphere relative shifts;

4. $\beta_{r e l}$ is the angle of the relative rotation around the EMC X axis $\left(0^{\circ}<\beta_{\text {rel }}<360^{\circ}\right)$.

These 12 parameters give a complete description of the hemisphere position for the first two layers. The choice of parametrization reflects our understanding of actual misalignment sources and their influence to the reconstruction.

Now let $\mathbf{p}_{\mathbf{0}}$ denote the point inside the aligned EMC hemisphere. The common EMC rotation can be represented by the matrix $\boldsymbol{T}_{\boldsymbol{z} \boldsymbol{y} \boldsymbol{z}}$ of the ZYZ rigid body rotation to the Euler angles relative to the TS center:

$$
\mathbf{T}_{\mathbf{z y z}}=\mathbf{R}_{\mathbf{z}}(\psi) \cdot \mathbf{R}_{\mathbf{y}}(\beta) \cdot \mathbf{R}_{\mathbf{z}}(\alpha-\psi) .
$$




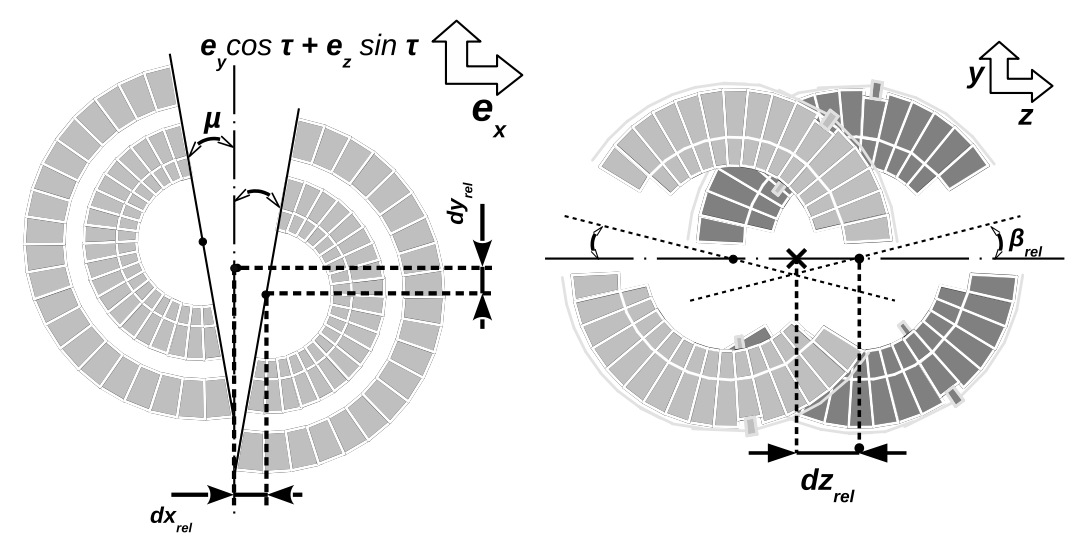

Figure 4: The relative parameters in the EMC reference system: left - the hemisphere separation and the relative shift along the $\mathrm{X}$ axis $\left(d x_{r e l}\right)$; right - the relative rotation around the $\mathrm{X}$ axis $\left(\beta_{\text {rel }}\right)$ and the relative shift along the $\mathrm{Z}$ axis $\left(d z_{\text {rel }}\right)$.

Hereinafter $\mathbf{R}_{\boldsymbol{a}}(\gamma)$ stands for the rotation around the $\boldsymbol{a}$ axis by an angle $\gamma$. The common shift vector is described as:

$$
\xi=\left(\begin{array}{l}
d x \\
d y \\
d z
\end{array}\right)
$$

The hemisphere separation can be described as a rotation around the axis perpendicular to the separation direction $(\tau)$ :

$$
\begin{aligned}
\mathbf{T}_{\mu} & =\mathbf{R}_{\mathbf{n}}(s \mu), \\
\mathbf{n} & =\left(\begin{array}{c}
0 \\
-\sin \tau \\
\cos \tau
\end{array}\right),
\end{aligned}
$$

where $s=\operatorname{sign}\left(\cos \varphi_{E M C}\right)$ is the sign of the hemisphere rotation or the shift. Similarly, the EMC hemisphere relative shift

$$
\xi_{\text {rel }}=s \cdot\left(\begin{array}{l}
d x_{r e l} \\
d y_{r e l} \\
d z_{r e l}
\end{array}\right),
$$

and the relative rotation around the $\mathrm{X}$ axis

$$
\mathbf{T}_{\beta_{\text {rel }}}=\mathbf{R}_{\mathbf{x}}\left(s \beta_{\text {rel }}\right) \text {. }
$$

The resulting transformation for $\mathbf{p}_{0}$ looks like

$$
\mathbf{p}_{\mathbf{1}}\left(\mathbf{p}_{\mathbf{0}} ; \boldsymbol{\eta}\right)=\mathbf{T}_{\mathbf{z y z}} \cdot\left(\mathbf{T}_{\mu} \cdot \mathbf{T}_{\beta_{\mathbf{r e l}}} \cdot \mathbf{p}_{\mathbf{0}}+\xi_{\text {rel }}\right)+\xi,
$$


where $\mathbf{p}_{\mathbf{1}}$ is the point of the misaligned EMC and $\boldsymbol{\eta}$ represents a set of 12 alignment parameters. As we take the hemispheres as rigid bodies the same transformation for counter centers and cluster positions can be used. The experimental distributions can be described by model functions for $\sin \left(\varphi_{T S, R}-\varphi_{E M C}\right)$ and $\theta_{T S, R}-\theta_{E M C}$ as

$$
\begin{aligned}
f_{\varphi}\left(\varphi_{0}, \theta_{0} ; \boldsymbol{\eta}\right) & =\sin \left(\varphi_{1}\left(\mathbf{p}_{\mathbf{0}} ; \boldsymbol{\eta}\right)-\varphi_{0}\right), \\
f_{\theta}\left(\varphi_{0}, \theta_{0} ; \boldsymbol{\eta}\right) & =\theta_{1}\left(\mathbf{p}_{\mathbf{0}} ; \boldsymbol{\eta}\right)-\theta_{0},
\end{aligned}
$$

where $\varphi_{0}, \theta_{0}$ are the $\mathbf{p}_{\mathbf{0}}$ azimuthal and polar angles, $\varphi_{1}, \theta_{1}$ are the $\mathbf{p}_{\mathbf{1}}$ azimuthal and polar angles. In data, the $\mathbf{p}_{\mathbf{1}}$ corresponds to the $\mathbf{p}_{\mathbf{R}}$ estimation from reconstructed TS data as described above.

For better convergence a number of optimizations is done during minimization. Parameter $\psi$ is periodic, its value poorly defined at small $\beta$. Therefore we replace the $(\psi, \beta)$ pair with the Cartesian parameters $\left(\beta_{1}, \beta_{2}\right)$ :

$$
\begin{aligned}
& \beta_{1}=\sin \beta \cos \psi \\
& \beta_{2}=\sin \beta \sin \psi
\end{aligned}
$$

An alternative parametrization is used also instead of the mixed angles and distance $\left(\tau, \mu, d x_{r e l}\right)$ parameters set. We use the distance only parameters $\left(d p_{1}, d p_{2}, d p_{3}\right)$ :

$$
\begin{aligned}
d x_{r e l} & =\frac{d p_{1}+d p_{2}+d p_{3}}{6} \\
\tau & =\arctan \frac{2 t}{d p_{1}-2 d x_{r e l}} \\
\mu & =\arcsin \frac{\sqrt{4 t^{2}+\left(d p_{1}-2 d x_{r e l}\right)^{2}}}{2 R_{1}}
\end{aligned}
$$

where $t=\frac{d p_{3}-d p_{2}}{2 \sqrt{3}}$. These parameters correspond to the distances between the hemispheres measured at the outer radius of the first supporting sphere $\left(R_{1}\right)$ at the angles $0^{\circ}, 120^{\circ}, 240^{\circ}$ from the vertical, respectively. These parameters have the same order of magnitude and type (units) and were measured directly early from deformation of pieces of some plastic substance inserted between the hemispheres. Also the negative values of these parameters' values indicates possible calibration or reconstruction errors which should be dealt before the calibration usage. Their non-negativeness means that the volumes of hemispheres do not overlap, it is important for reconstruction and MC simulation.

\section{Procedure of retrieving alignment parameter values}

The procedure of retrieving alignment parameter values includes several steps. First of all, $e^{+} e^{-} \rightarrow e^{+} e^{-}$events are selected using the following criteria: 
1. no reconstructed photons and exactly two charged tracks, both with the shift along beam $|z|<10 \mathrm{~cm}$ and the impact parameter $|\rho|<1 \mathrm{~cm}$;

2. the energy deposition in the EMC for each particle $0.8 E_{\text {beam }}<E_{1,2}<$ $1.1 E_{\text {beam }},(i=1,2)$;

3. acollinearity in the TS in the azimuth direction $\left|180^{\circ}-\right| \varphi_{1}-\varphi_{2}||<10^{\circ}$;

4. muon veto signal absence.

The second condition effectively suppresses influence of not operating EMC counters.

The next step is to fill the $2 \mathrm{D}$ profiles of $\sin \left(\varphi_{T S, R}-\varphi_{E M C}\right)$ and $\theta_{T S, R}-$ $\theta_{E M C}$ depending of the azimuthal angle range from $0^{\circ}$ to $360^{\circ}$ and the polar angle range from $45^{\circ}$ to $135^{\circ}$ using data events reconstructed in the EMC and in the TS. The size of the polar angle bin is set to the counter angle size, while the size of the azimuthal angle bin is chosen to fit a whole number of the TS cell halves and the EMC counter halves. This is done to minimize effects from local non-uniformity in reconstructed angles $([13])$. The total number of bins is 160 $\left(16 \varphi_{E M C} \times 10 \theta_{E M C}\right)$. Using finer granularity for binned fit or using unbinned fit would require to take into account exact shape of the local non-uniformity and would unnecessary complicate the procedure.

The fit function is constructed as a sum of the squared differences between the measured angle residuals and the expected ones parametrized with the model functions $f_{\varphi}, f_{\theta}(\mathrm{Eq} 6)$ scaled by the measurement error:

$$
\begin{aligned}
\chi^{2}(\boldsymbol{\eta}) & =\sum_{i} \frac{\left(\sin \left(\varphi_{T S, R}-\varphi_{E M C}\right)_{i}-f_{\varphi}\left(\varphi_{E M C_{i}}, \theta_{E M C_{i}} ; \boldsymbol{\eta}\right)\right)^{2}}{\sigma_{\varphi_{i}}^{2}} \\
& +\sum_{i} \frac{\left(\left(\theta_{T S, R}-\theta_{E M C}\right)_{i}-f_{\theta}\left(\varphi_{E M C_{i}}, \theta_{E M C_{i}} ; \boldsymbol{\eta}\right)\right)^{2}}{\sigma_{\theta_{i}}^{2}}
\end{aligned}
$$

where the subscript index $i$ runs over all the spatial 160 bins and denotes averaging over all the selected events in the current bin, $\sigma_{\varphi_{i}}$ and $\sigma_{\theta_{i}}$ are the measurement errors. Each error includes both a statistical and systematic component:

$$
\sigma^{2}=\sigma_{\text {stat }}^{2}+\sigma_{\text {sys }}^{2} .
$$

The systematic uncertainty is attributed to the non-uniformity in the EMC spatial reconstruction and shortcoming of the model where we ignore 3rd layer contribution. It is expected to be the same for polar and azimuth direction and estimated from the data to be $0.2^{\circ}$. The fit function doesn't include the correlation term between azimuthal and polar parts because they were measured using independent detector elements. The minimization is performed using the TMinuit class from the ROOT framework [14]. The fit process includes the following stages of the $\chi^{2}$ function minimization. At the first stage all parameters are set to be zero and all of them except $\alpha$ are fixed. At the next stages the rest of the parameters become free in the following order: $(d x, d y) ;\left(d z, \beta_{1}, \beta_{2}\right)$; $\left(d p_{1}, d p_{2}, d p_{3}\right) ;\left(d y_{r e l}, d z_{r e l}\right) ;\left(\beta_{r e l}\right)$. 

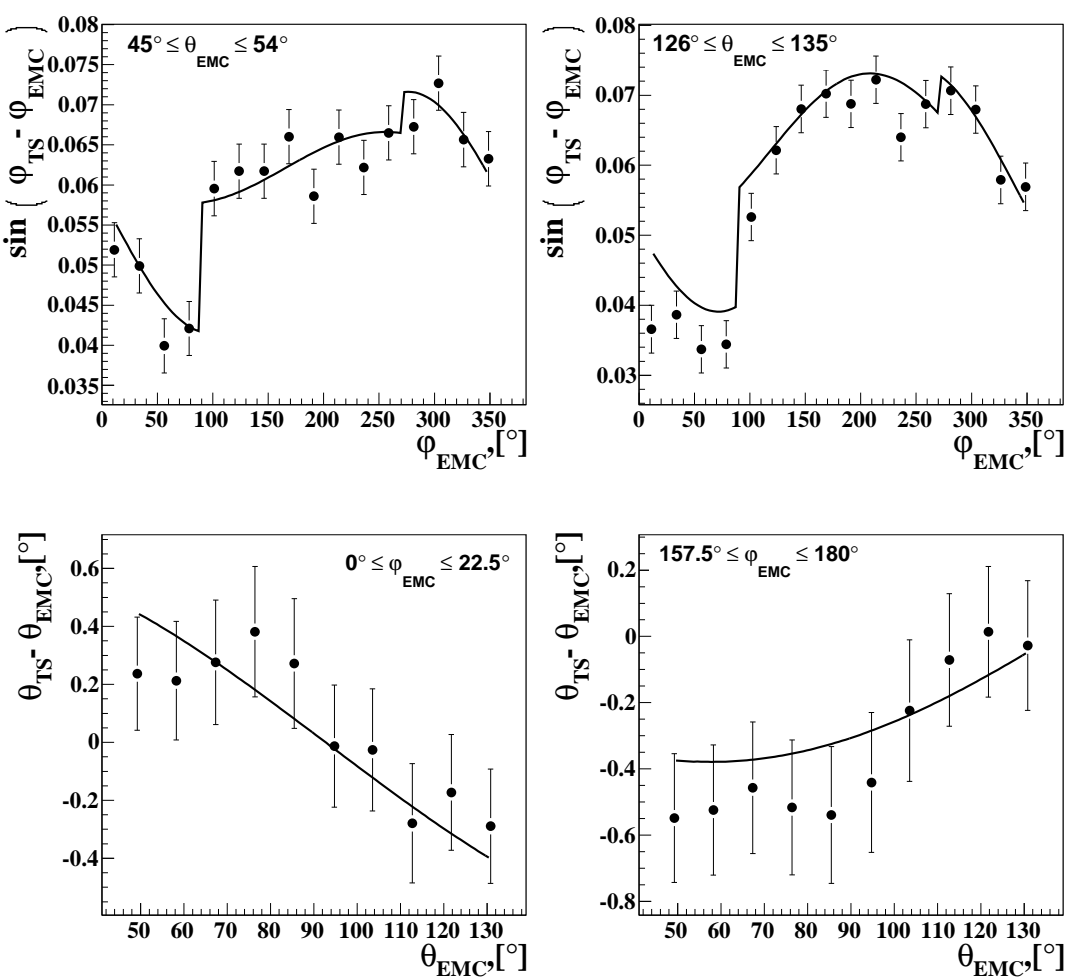

Figure 5: The results of the fit to data at $E_{\text {beam }}=612.5 \mathrm{MeV}$. The solid lines represent the fitted functions. Points with errors show the data distribution. The errors include the statistical and systematic uncertainties.

The results of a typical fit are shown in Fig.5. The discontinuities of the fitted curve is explained by changing the sign of the relative translation and the relative rotation for EMC counters close to the vertical direction.

\section{Application of the obtained parameters}

The obtained alignment parameter values are used both in event reconstruction and in MC simulation. Data processing is performed with the SND framework [15]. Being the main part of offline SND software this framework supports reconstruction, analysis and MC simulation.

The role of the geometric database in the framework belongs to an object of the GeoEmcDBase class from the framework GeoDesc package. Methods of this class transform an index of an EMC counter into geometric coordinates (Cartesian, cylindrical or polar), which are used then in reconstruction. Other methods of this class perform inverse transformation. At the beginning parameters of the aligned EMC are calculated and then they are corrected using 
geometric calibration results. Further reconstruction uses these corrected EMC element coordinates.

During reconstruction the transformation inverse to Eq[5 is used for transforming a point of the misaligned EMC to a point of the aligned EMC:

$$
p_{0}=\mathbf{T}_{\mu}^{T} \cdot \mathbf{T}_{\beta_{r e l}}^{T} \cdot\left(\mathbf{T}_{\mathbf{z y z}}^{\mathbf{T}} \cdot\left(\mathbf{p}_{\mathbf{1}}-\xi\right)-\xi_{\text {rel }}\right) .
$$

The alignment parameter values are determined only for the first two EMC layers, so Eq[5 and Eq9 can be safely used for clusters only in these layers. To avoid overlap of the two third layer hemispheres, in the case if the following condition is true (Eq, 10):

$$
d x_{r e l}-R_{p_{3}} \sin \mu<0
$$

clusters of this layer are corrected using the same formulas but with $\mu$ substituted by $\mu_{3}$ :

$$
\mu_{3}=\arcsin \frac{d x_{r e l}}{R_{p_{3}}},
$$

where $R_{p_{3}}$ is the outer radius of the second supporting sphere.

For realistic MC simulation volumes representing the EMC hemispheres are placed in the world volume taking into account the obtained alignment parameter values using Eq5. This is performed by applying corresponding transformation to the nested volumes. Due to the hierarchy of the volumes there no need to place individual counters. The third layer hemispheres are placed taking into account the above considerations (Eq10) in order to perform correct MC simulation.

\section{Results and discussion}

The alignment algorithm is validated using MC simulation, that takes into account the alignment parameters to reproduce the EMC displacement effects observed in data. To improve the quality of the simulation, non-operating EMC counters are masked. To take into account beam-generated spurious hits in the detector in MC, special events recorded during experiment with a random trigger are merged with the simulated events [16].

To validate the algorithm, $100 \mathrm{MC}$ simulation samples are produced with different sets of the alignment parameters evenly distributed over the expected range. Used statistics of 50000 events per sample is compatible with the statistics used for one calibration for data. The biases and spreads of the parameters, estimated as the means and RMSs of the differences between the values obtained from the fit and the true MC values, are listed in Table 1 The largest biases are observed for $\alpha$ and $d y_{r e l}$. These parameters also have the largest negative correlation $(-0.85)$ between them because the relative shift along the $\mathrm{Y}$ axis of the EMC halves in opposite directions adds an effective bias of the rotation angle around the $\mathrm{Z}$ axis. The biases do not exceed $0.2^{\circ}$ for angles and $0.06 \mathrm{~cm}$ for 


\begin{tabular}{|c|c|c|c|c|c|}
\hline EMC & bias & spread & Halves & bias & spread \\
\hline \hline$\alpha,^{\circ}$ & -0.13 & 0.02 & $\mu,^{\circ}$ & $-110^{-4}$ & $310^{-4}$ \\
\hline$\beta_{1},{ }^{\circ}$ & -0.007 & 0.023 & $d x_{r e l}, \mathrm{~cm}$ & -0.021 & 0.008 \\
\hline$\beta_{2},{ }^{\circ}$ & -0.006 & 0.017 & $d p_{1}, \mathrm{~cm}$ & -0.045 & 0.031 \\
\hline$d x, \mathrm{~cm}$ & -0.005 & 0.006 & $d p_{2}, \mathrm{~cm}$ & -0.030 & 0.030 \\
\hline$d y, \mathrm{~cm}$ & -0.005 & 0.012 & $d p_{3}, \mathrm{~cm}$ & -0.053 & 0.036 \\
\hline$d z, \mathrm{~cm}$ & 0.012 & 0.021 & $\beta_{\text {rel }},{ }^{\circ}$ & $-110^{-4}$ & $510^{-4}$ \\
\hline & & & $d y_{r e l}, \mathrm{~cm}$ & 0.052 & 0.018 \\
\hline & & & $d z_{r e l}, \mathrm{~cm}$ & -0.005 & 0.018 \\
\hline
\end{tabular}

Table 1: The biases and spreads of the alignment parameters obtained using MC simulation.
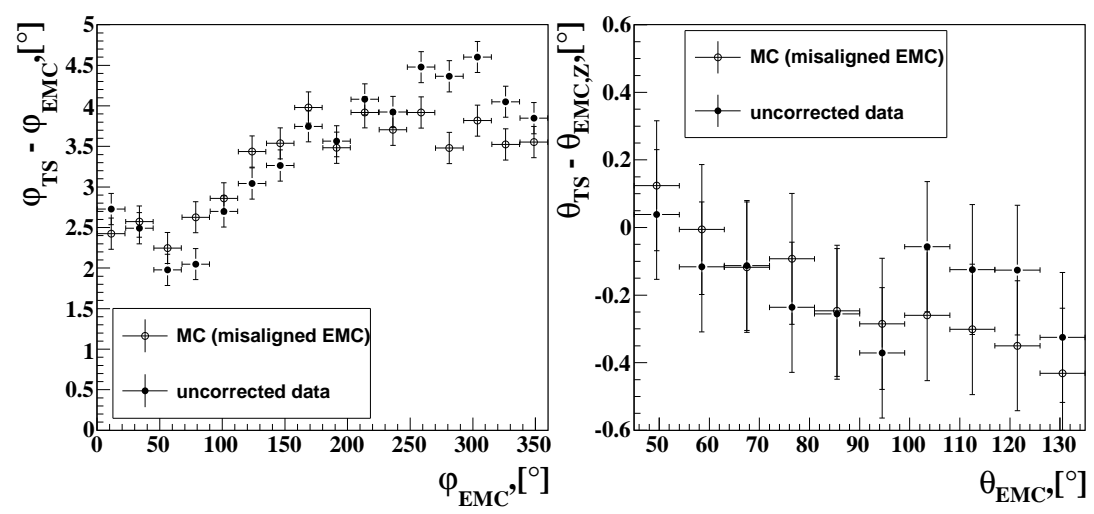

Figure 6: Comparison of the difference between the angles reconstructed in the EMC and in the TS for $e^{+} e^{-} \rightarrow e^{+} e^{-}$events in uncorrected data (filled circle) and realistic MC simulation (empty circle) at $E_{\text {beam }}=612.5 \mathrm{MeV}$. In calculation of $\theta_{E M C, z}$ the shift of the event vertex along the $\mathrm{Z}$ axis is taken into account. The errors include the statistical and systematic uncertainties.

translations and are too small to lead to observable shifts in reconstructed photon parameters. Therefore, we conclude that the true and measured parameter values are in reasonable agreement.

Comparison of the described above realistic MC simulation with the uncorrected data, shown in Fig 6, demonstrates satisfactory agreement.

The procedure described in Sec 3 is applied to about 2000 runs during 20102011 recorded with an integrated luminosity of $25.3 p b^{-1}$. In Fig:7 the dependence of obtained alignment parameters on time is shown. The value of $\alpha$ was stable during this period, while the value of $d x$ changed several times due to disassembling and reassembling the detector. One of these mechanical interruptions also changed the angle between the TS and the EMC axes $(\psi)$. We do not see steady changes in the EMC geometry parameters. This supports our assumption on these changes origin. It is also possible to use these graphs for quality control: steady changes in parameter values indicate incorrect event reconstruction in the TS or in the EMC. The obtained relative shift along the 

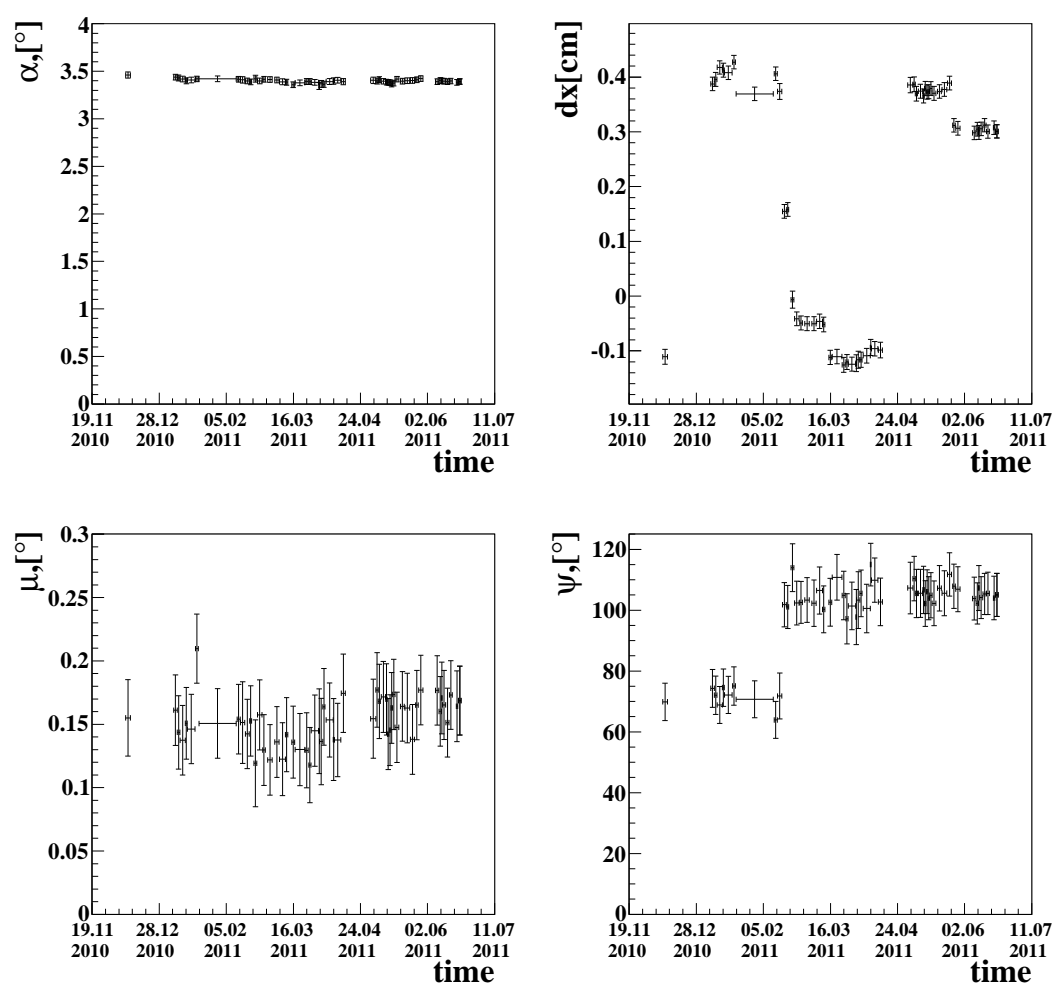

Figure 7: The time evolution of the values of the parameters $\alpha, d x, \mu, \psi$.

$\mathrm{X}$ axis and the relative rotation around the $\mathrm{Z}$ axis are close to measured with instrumental methods.

The results of the geometric alignment $\left(E_{\text {beam }}=612.5 \mathrm{MeV}\right)$ for $e^{+} e^{-} \rightarrow$ $e^{+} e^{-}$events are shown in Fig, 8 . Taking into account the relative rotation angle of the EMC halves can eliminate its systematic contribution to the difference between the angles of photons traversing the different EMC halves. This difference can be observed in the acollinearity distribution for the $e^{+} e^{-} \rightarrow 2 \gamma$ events (Fig 9) selected with the following conditions:

1. exactly two reconstructed photons and zero charged tracks;

2. the energy deposition in the EMC for each particle $E_{1,2}>0.7 E_{\text {beam }}$;

3. difference between particle energies $\left|E_{1}-E_{2}\right|<150 \mathrm{MeV}$;

4. muon veto signal absence;

5. $36^{\circ}<\theta_{E M C_{1,2}}<144^{\circ}$.

Figure 9 demonstrates 16\% improvement in the EMC angular resolution due to the correction.

To study the influence of the geometric calibration on the reconstruction of events containing both charged particles and photons (mixed charge). The 

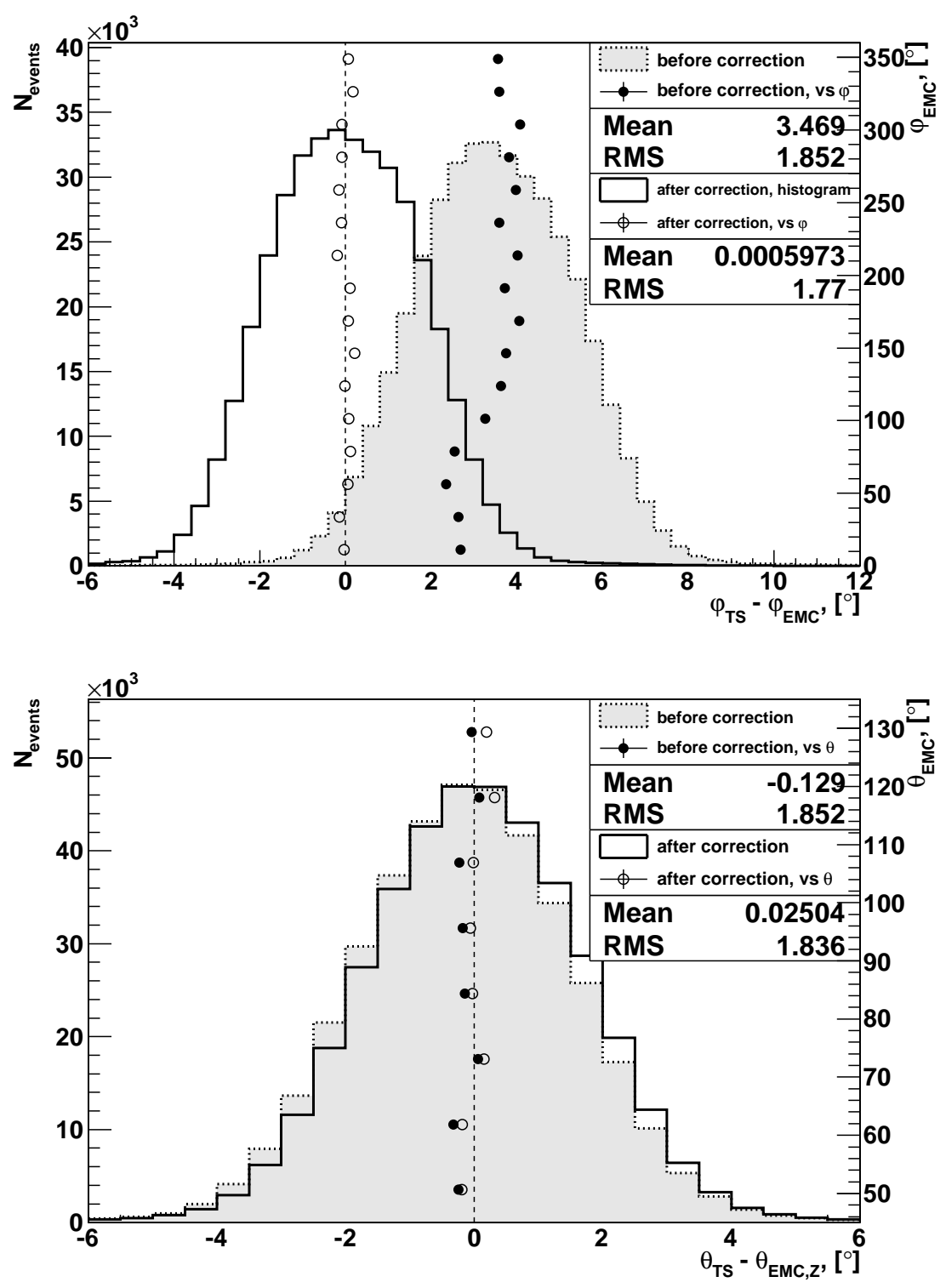

Figure 8: Results of the geometric correction for $e^{+} e^{-} \rightarrow e^{+} e^{-}$data at $E_{\text {beam }}=612.5 \mathrm{MeV}$. The left scale and filled and opened histograms represent the difference between angles reconstructed in the EMC and in the TS before and after the corrections respectively. The right scale and filled and opened circles represent the same difference as functions of corresponding EMC angles before and after the corrections respectively. In calculation of $\theta_{E M C, z}$ the shift of the event vertex along the $\mathrm{Z}$ axis is taken into account. 


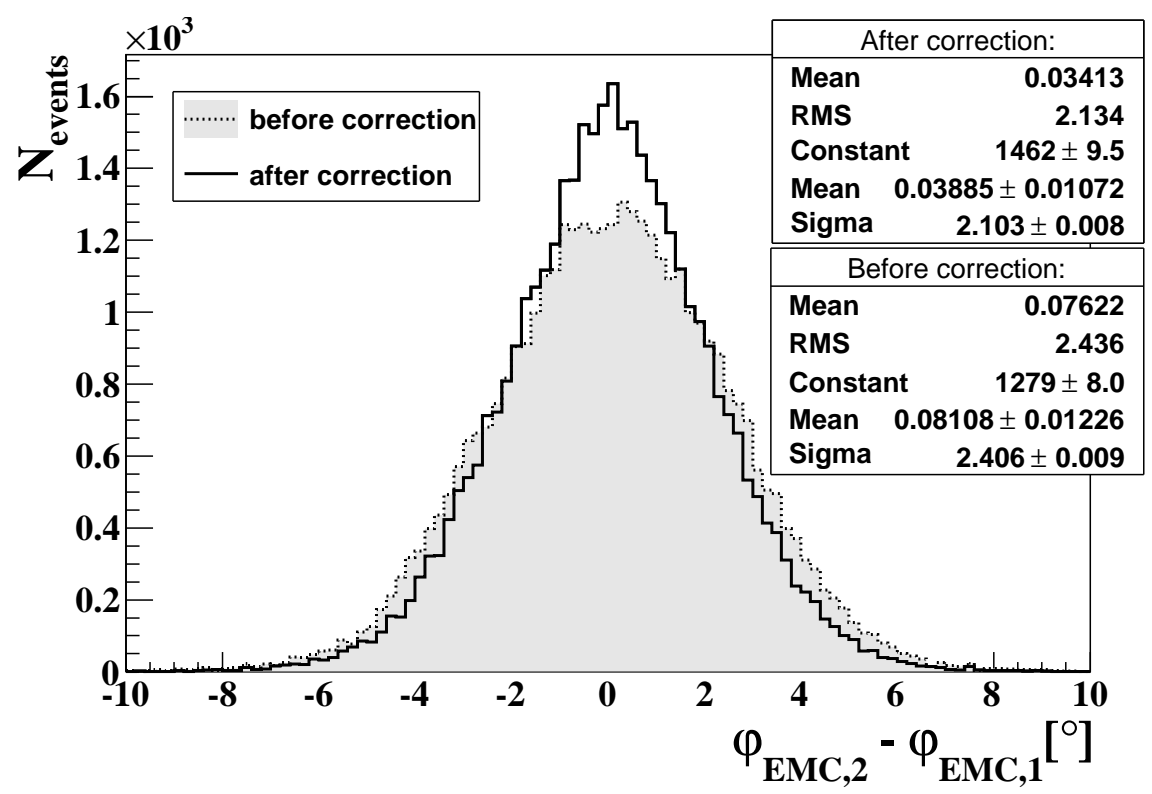

Figure 9: The result of the geometric correction for $e^{+} e^{-} \rightarrow 2 \gamma$ events at $E_{\text {beam }}=612.5$ $\mathrm{MeV}$. Filled and opened histograms represent the difference between angles reconstructed in the EMC and in the TS before and after the corrections respectively.

process $e^{+} e^{-} \rightarrow \pi^{+} \pi^{-} \pi^{0}$ is used. The candidate events from the energy range near the $\phi(1020)$ resonance are selected using the following conditions:

1. exactly two charged particles, two neutral particles;

2. $36^{\circ}<\theta_{\operatorname{chr} 1,2}<144^{\circ}$;

3. $\left|180^{\circ}-\right| \varphi_{\text {chr } 1}-\varphi_{\text {chr } 2}||>10^{\circ}$;

4. $\arccos \left(n_{\operatorname{chr} 1} \cdot n_{\operatorname{chr} 2}\right)<147^{\circ}$;

5. the energy deposition form photons $E_{N 1}+E_{N 2}>100 \mathrm{MeV}$.

Then a kinematic fit is performed with the requirements of energy and momentum conservation and without the $\pi^{0}$ mass constraint. The fit uses photon angles and energies and only angles for charged particles. It should be noted that our detector does not measure momenta of charged particles. The corrections lead to significant improvement of the fit quality (Fig[10). It should be noted that because non-Gaussian distribution of measured parameters fit quality parameter not necessary follows the $\chi^{2}$ distribution.

\section{Conclusions}

We have developed and implemented the geometric calibration procedure for the SND detector electromagnetic calorimeter. The procedure is validated using MC simulation. It is shown that most of the alignment parameters values stay stable during the data taking. Some of them slightly changes due to disassembling and reassembling the detector. 


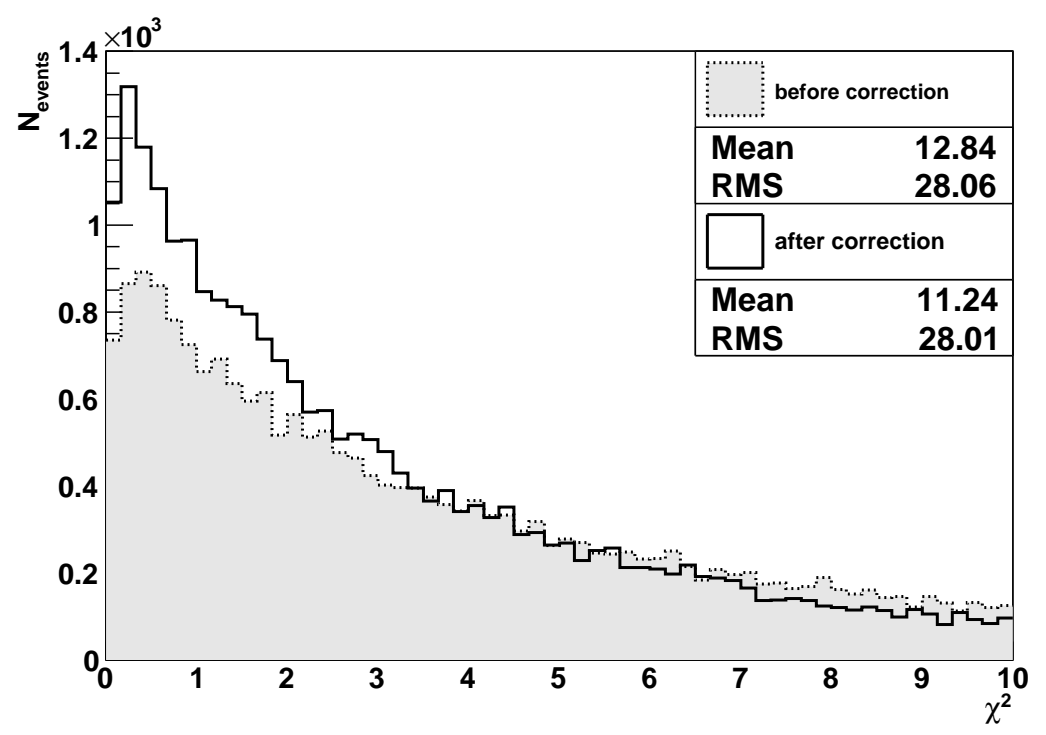

Figure 10: The kinematic fit quality parameter for $e^{+} e^{-} \rightarrow \pi^{+} \pi^{-} \pi^{0}$ events. Filled and opened histograms represent the difference between angles reconstructed in the EMC and in the TS before and after the corrections respectively.

The procedure allows us to reduce the difference between the angles measured in the TS and the EMC. The common bias in the azimuthal angle about $3^{\circ}$ and its irregularity of about $1^{\circ}$ are removed. The angular correction allows us to improve the kinematic fit quality for mixed charge events (the $\chi^{2}$ mean value decreases by $10-15 \%$ ).

The results of the geometric calibration are used in the data analysis and MC simulation [17, 18, 19]. We think that the ideas implemented in the calibration procedure can be useful in other experiments with heterogeneous detectors as well.

\section{Acknowledgments}

This work was supported by the Russian Science Foundation [grant number 14-50-00080].

\section{References}

[1] M. N. Achasov, et al., Spherical neutral detector for VEPP-2M collider, Nucl. Instrum. Meth. A449 (2000) 125-139. arXiv:hep-ex/9909015, doi:10.1016/S0168-9002(99)01302-9.

[2] G. N. Abramov, et al., SND upgrade, eConf C010430 (2001) T10. arXiv:hep-ex/0105093 
[3] V. M. Aulchenko, et al., SND tracking system: Tests with cosmic muons, Nucl. Instrum. Meth. A598 (2009) 102-104. doi:10.1016/j.nima.2008.08.099

[4] B. I. Khazin, Detectors and physics at VEPP-2000, Nucl. Instrum. Meth. A623 (2010) 353-355. doi:10.1016/j.nima.2010.02.246

[5] S. Blusk, O. Buchmuller, A. Jacholkowski, T. Ruf, J. Schieck, S. Viret (Eds.),

Proceedings, first LHC Detector Alignment Workshop, CERN, Geneva, Switzerland, 4-6 September 2006 2007.

URL http://cdsweb.cern.ch/record/970621

[6] G. Aad, et al., Electron performance measurements with the ATLAS detector using the 2010 LHC proton-proton collision data, Eur. Phys. J. C72 (2012) 1909. arXiv:1110.3174, doi:10.1140/epjc/s10052-012-1909-1.

[7] V. V. Anashin, et al., The KEDR detector, Phys. Part. Nucl. 44 (2013) 657-702. doi:10.1134/S1063779613040035

[8] V. M. Aulchenko, et al., CsI calorimeter of the CMD-3 detector, JINST 10 (10) (2015) P10006. doi:10.1088/1748-0221/10/10/P10006

[9] J. M. Bauer, The BaBar electromagnetic calorimeter: Status and performance improvements, in: 2005 IEEE Nuclear Science Symposium and Medical Imaging Conference El Conquistador Resort, Puerto Rico, October23-29, 2005, Vol. 2, 2006, pp. 1038-1042. arXiv:physics/0601138, doi:10.1109/NSSMIC.2005.1596430

[10] K. A. Olive, et al., Review of Particle Physics, Chin. Phys. C38 (2014) 090001. doi:10.1088/1674-1137/38/9/090001.

[11] V. M. Aulchenko, et al., SND tracking system: Tests with cosmic muons, Nucl. Instrum. Meth. A598 (2009) 102-104. doi:10.1016/j.nima.2008.08.099

[12] A. Yu. Barnyakov, et al., Particle identification system based on dense aerogel, Nucl. Instrum. Meth. A732 (2013) 330-332. doi:10.1016/j.nima.2013.05.075

[13] M. Bekishev, V. Ivanchenko, A method of electromagnetic shower identification and measuring of its posi Nuclear Instruments and Methods in Physics Research Section A: Accelerators, Spectrometers, Detectors and Associated Equipment 361 (1) (1995) 138 - 148. doi:http://dx.doi.org/10.1016/0168-9002(95)00027-5. URL http://www.sciencedirect.com/science/article/pii/0168900295000275

[14] R. Brun, F. Rademakers, ROOT: An object oriented data analysis framework, Nucl. Instrum. Meth. A389 (1997) 81-86. doi:10.1016/S0168-9002(97)00048-X. URL http://root.cern.ch 
[15] A. D. Bukin, et al., SND off-line framework, in: H. S. Chen, et al. (Eds.), CHEP 2001: Proceedings of the International Conference on Computing in High Energy and Nuclear Physics September 3-7, 2001 Beijing, P.R. China, Science Press, New York, 2001, pp. 145-148.

[16] M. N. Achasov, et al., Study of $e^{+} e^{-} \rightarrow \omega \pi^{0} \rightarrow \pi^{0} \pi^{0} \gamma$ in the energy range $1.05-2.00 \mathrm{GeV}$ with SND, Phys. Rev. D88 (5) (2013) 054013. arXiv:1303.5198, doi:10.1103/PhysRevD.88.054013.

[17] M. N. Achasov, et al., Search for the $\eta^{\prime} \rightarrow e^{+} e^{-}$decay with the SND detector, Phys. Rev. D91 (2015) 092010. arXiv:1504.01245, doi:10.1103/PhysRevD.91.092010

[18] M. N. Achasov, et al., Measurement of the $K_{L}$ nuclear interaction length in the $\mathrm{NaI}(\mathrm{Tl})$ calorimeter, JINST 10 (2015) P09006. arXiv:1509.01918, doi:10.1088/1748-0221/10/09/P09006.

[19] M. N. Achasov, et al., Recent results from SND detector, AIP Conf. Proc. 1735 (2016) 030009. doi:10.1063/1.4949392. 


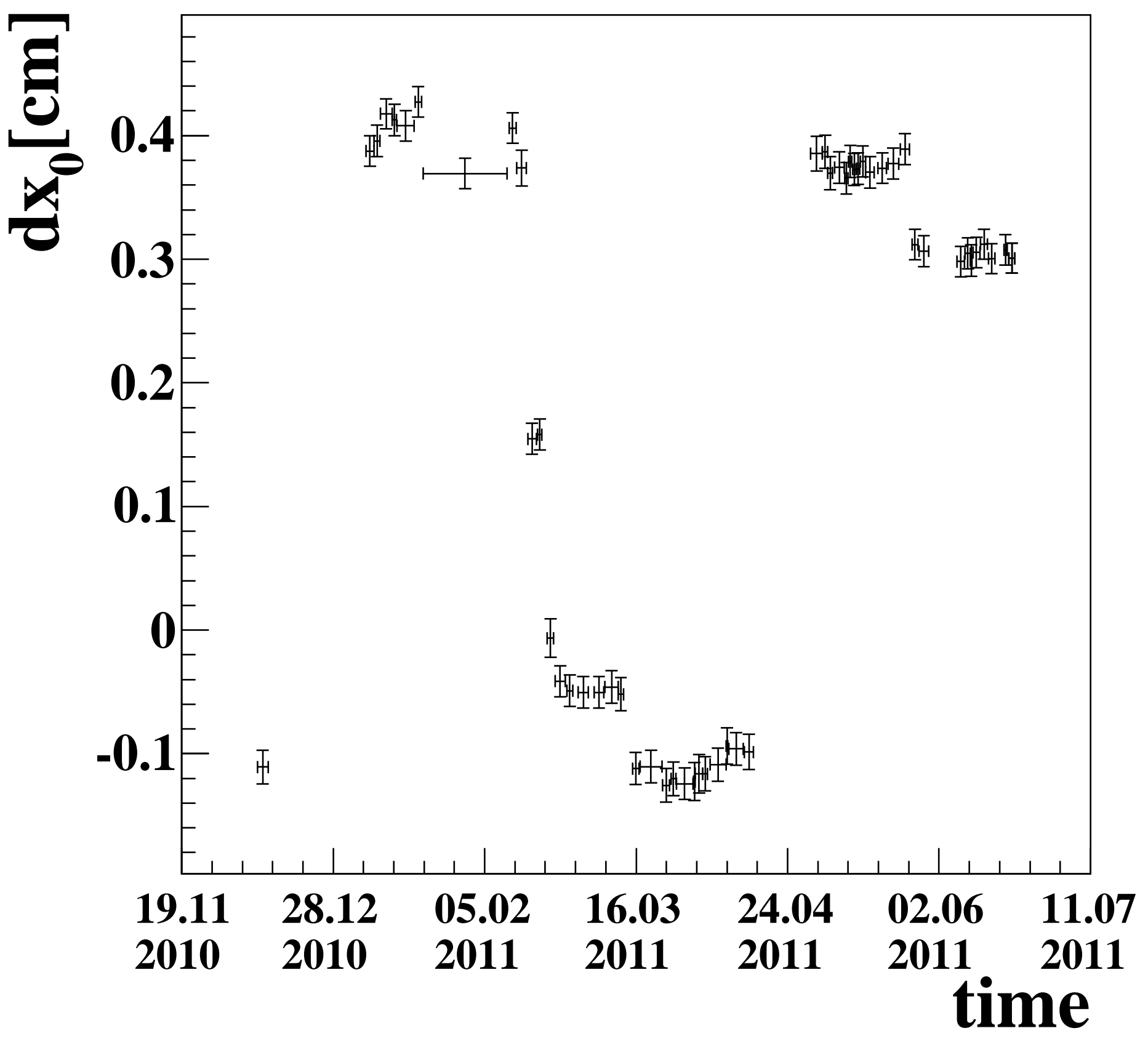




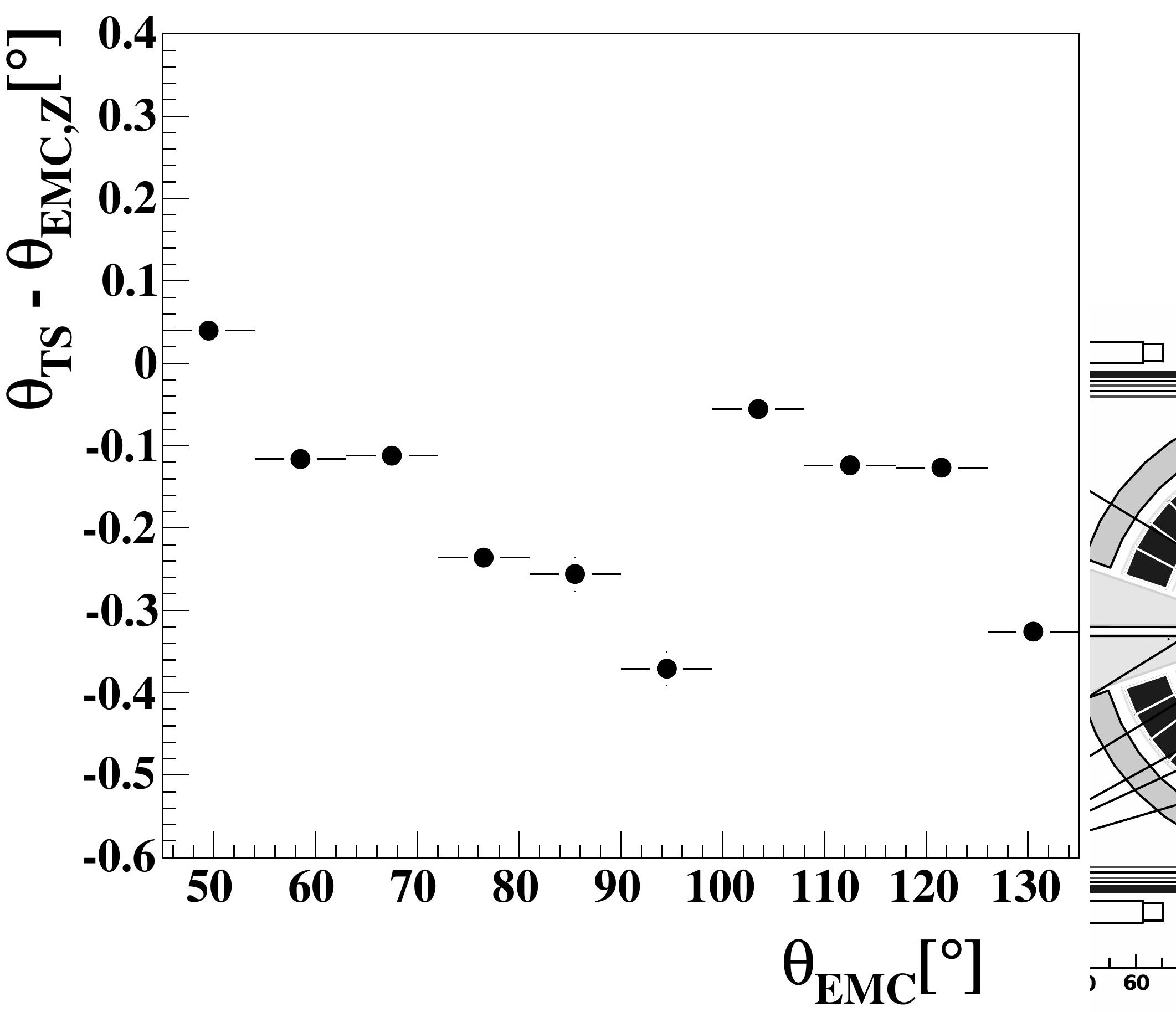




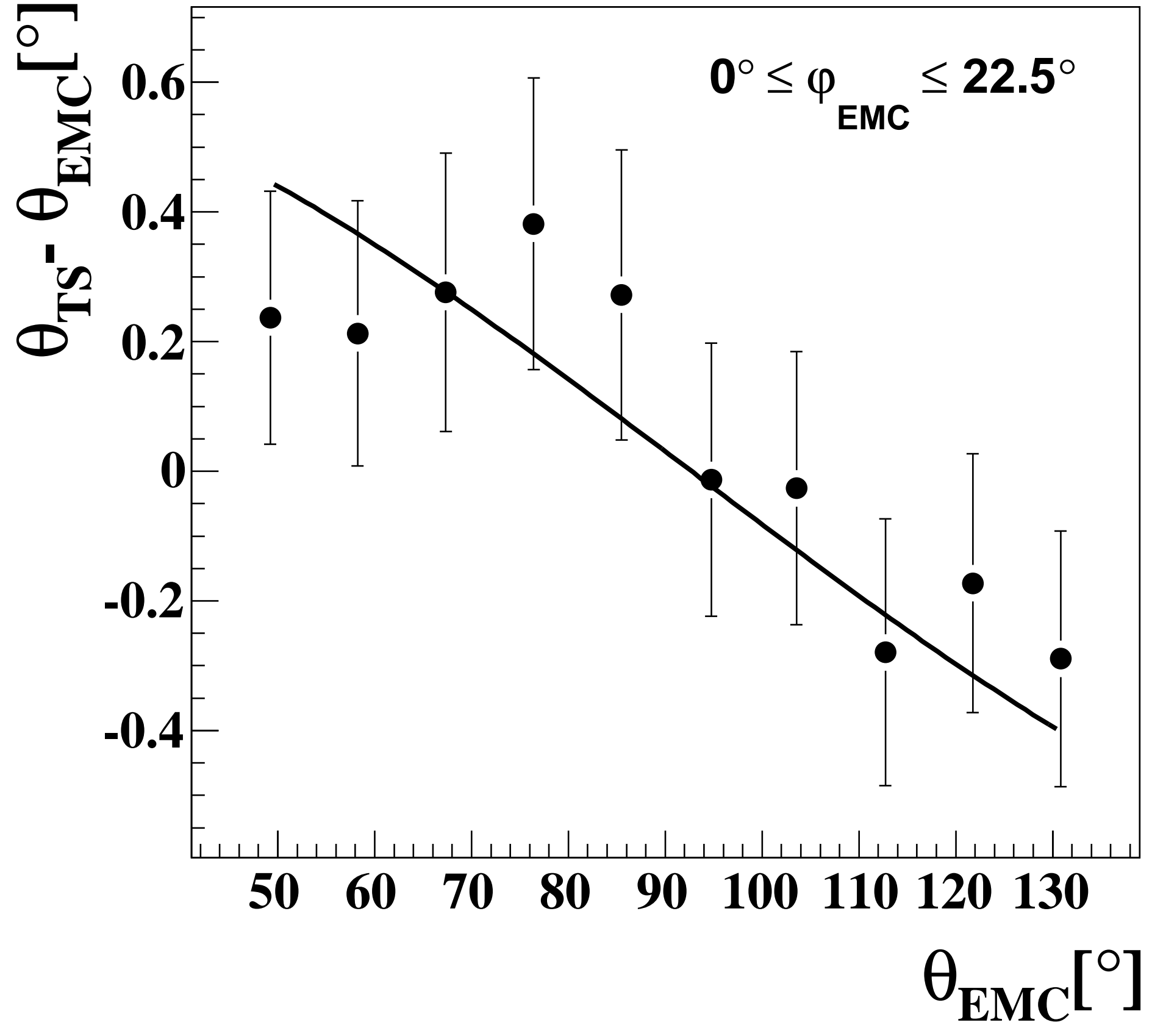




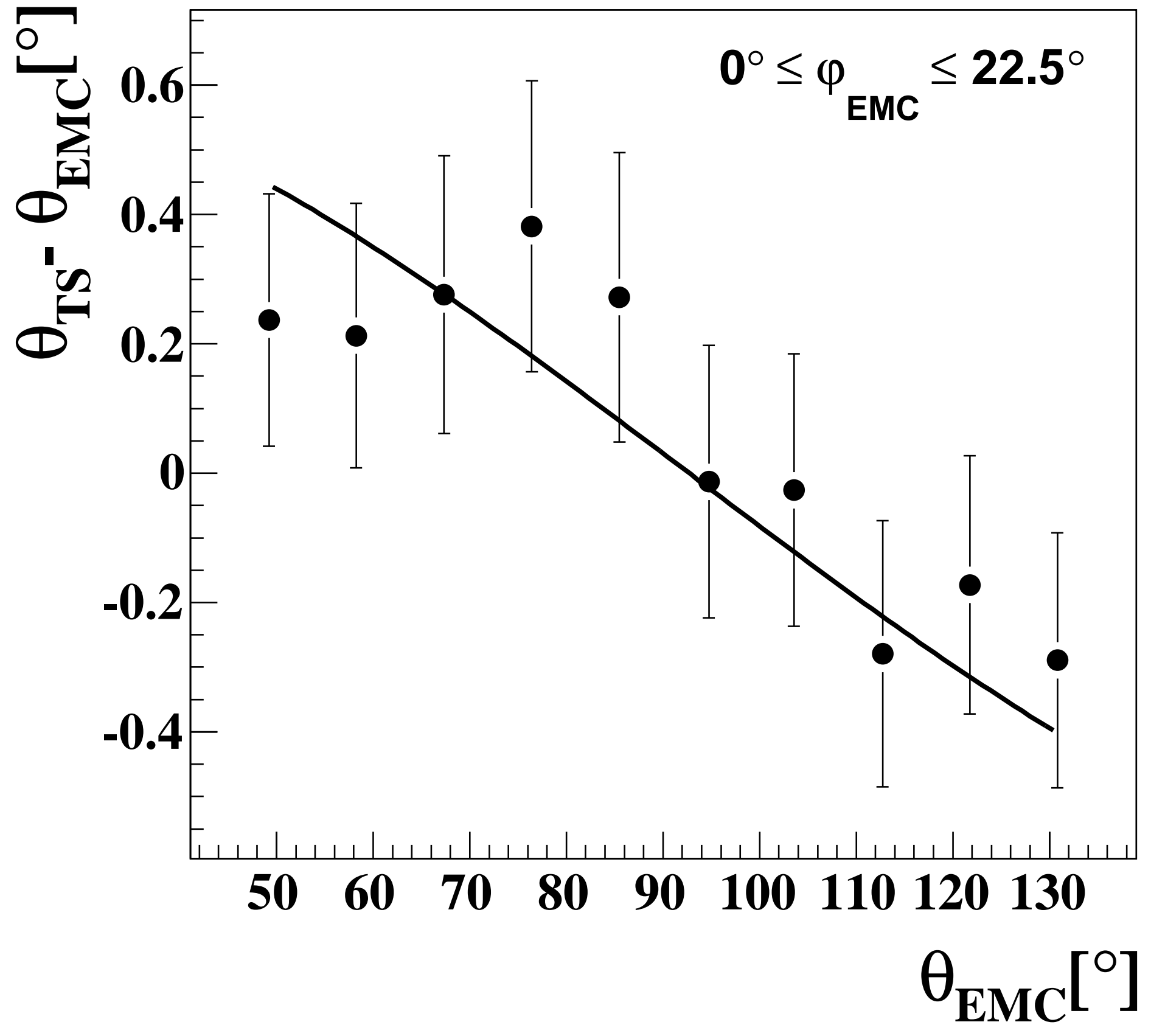




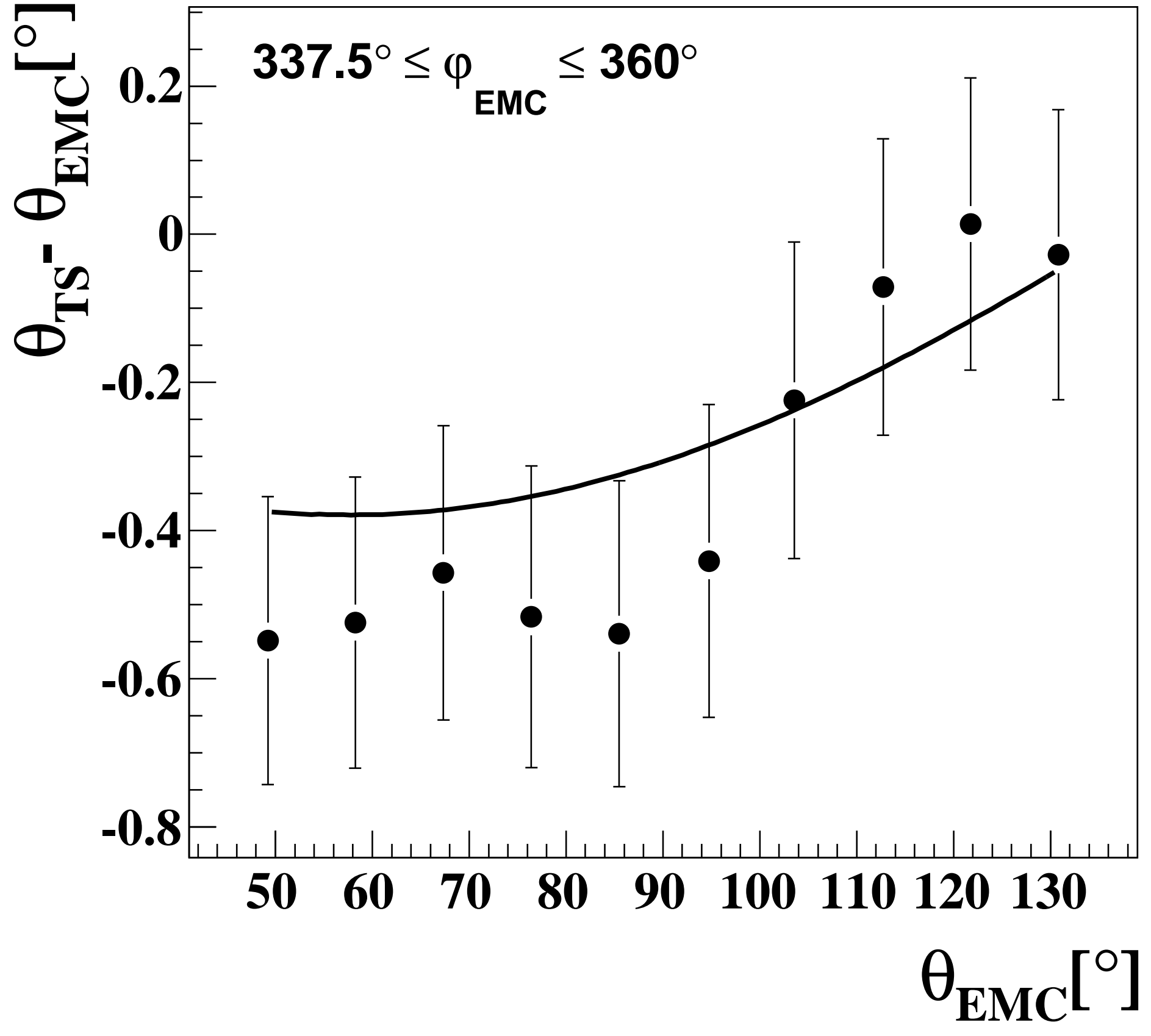

